Genome Insight Plant Genetics

\title{
Chloroplast genome characteristics and phylogenetic analysis of the medicinal plant Blumea balsamifera (L.) DC
}

\author{
Chao Zhao ${ }^{1}$ (D), Wenfen $\mathrm{Xu}^{1}$ (D), Yuan Huang ${ }^{1}$ (D), Qingwen Sun ${ }^{1}$, Bo Wang ${ }^{1}$, Chunlin Chen $^{1}$ and Qiyu Chen ${ }^{1}$ \\ ${ }^{I}$ Guizhou University of Traditional Chinese Medicine, College of Pharmacy, Guiyang, China.
}

\begin{abstract}
Blumea balsamifera (L.) DC., a medicinal plant with high economic value in the Asteraceae family, is widely distributed in China and Southeast Asia. However, studies on the population structure or phylogenetic relationships with other related species are rare owing to the lack of genome information. In this study, through high-throughput sequencing, we found that the chloroplast genome of $B$. balsamifera was $151,170 \mathrm{bp}$ in length, with a pair of inverted repeat regions (IRa and IRb) comprising 24,982 bp, a large single-copy (LSC) region comprising $82,740 \mathrm{bp}$, and a small single-copy (SSC) region comprising $18,466 \mathrm{bp}$. A total of 130 genes were identified in the chloroplast genome of B. balsamifera, including 85 protein-coding, 37 transfer RNA, and 8 ribosomal RNA genes; furthermore, sequence analysis identified 53 simple sequence repeats. Whole chloroplast genome comparison indicated that the inverted regions (IR) were more conserved than large single-copy and SSC regions. Phylogenetic analysis showed that $B$. balsamifera is closely related to Pluchea indica. Conclusively, the chloroplast genome of $B$. balsamifera was helpful for species identification and analysis of the genetic diversity and evolution in the genus Blumea and family Asteraceae.
\end{abstract}

Keywords: Blumea balsamifera, chloroplast genome, codon usage, repeat sequence, phylogenetic analysis.

Received: April 02, 2021; Accepted: September 13, 2021.

\section{Introduction}

Blumea balsamifera (L.) DC. is a perennial herb or subshrub that belongs to the family Asteraceae and is mainly distributed in China, India, Thailand, and the Philippines (Chinese Academy of Sciences editorial commission of the flora, 1988). As a traditional ethnic Miao's medicinal plant in China, B. balsamifera is commonly called Ai-Na-Xiang and Da-Feng-Ai and is extensively used to treat eczema, dermatitis, rheumatism, and wind syndrome of the head (Dewen, 2005). In the Philippines, this plant is called Sambong, a leading prescribed medicine for diuresis and antiurolithiasis (Toralba et al., 2015). Recent studies have demonstrated that this plant possesses several pharmacological activities, such as antitumor (Hasegawa et al., 2006) and anti-inflammatory (Sakee et al., 2011). Additionally, because of its strong aromatic properties, the leaves of the plant are used as flavoring ingredients and tea (Xu et al., 2012), which have great economic value and attract the attention of researchers.

Asteraceae is the largest family of flowering plants with numerous species, including 1,479 genera and 21,105 species. It is widely distributed in the world except the Antarctic region (Dempewolf et al., 2008). Blumea is a highly complex genus in the Asteraceae family. It was first placed in the tribe Astereae and later moved to the tribe Inuleae owing to the characteristics of anthers. In 1989, Anderberg separated the tribe Gnaphalieae from the tribe Inuleae based on the morphological characteristics of plants and confirmed the accuracy of the classification based on the results of $n d h F$ sequencing analysis (Anderberg et al.,

Send correspondence to Wenfen Xu. Guizhou University of Traditional Chinese Medicine, College of Pharmacy, Guiyang, 550025, Guizhou, China. E-mail: wenfenxu@126.com.
1991, 2005). However, the above classification based on plant morphology and short DNA sequences has limitations; therefore, more information needs to be collected for analysis and discussion. Chloroplasts are important organelles in green plants which mainly participate in protein, pigment, and starch biosynthesis (Rodriguez-Ezpeleta et al., 2005). Additionally, the chloroplast genome has fewer nucleotide substitutions and rearrangement of genome structure than the nuclear genome (Smith, 2015). The genome size, content, and structure are more conserved in the chloroplast genome than in the nuclear genome (Wicke et al., 2011). Therefore, it becomes an ideal model for studying genome evolution, phylogenetic analysis, and species identification in complex angiosperm families. However, the chloroplast genome of Blumea has not yet been reported, which is inconducive to further study on the evolutionary history and status of Blumea.

Therefore, this study adopted Illumina sequencing technology to sequence the whole chloroplast (cp) genome of B. balsamifera and compared it with other available Asteraceae species to explore their genetic divergence, genetic structural characteristics, and phylogenetic relationships. Thus, our study provides valuable information for elucidating the evolution of B. balsamifera, developing molecular markers, and revealing phylogenetic relationships in the Asteraceae family.

\section{Material and Methods}

\section{Plant material, DNA extraction and sequencing}

Fresh leaves of $B$. balsamifera were collected from Hongshuihe Town, Luodian County, Guizhou Province, China $\left(25^{\circ} 09^{\prime} 56.8^{\prime \prime} \mathrm{N}, 106^{\circ} 37^{\prime} 24.1^{\prime \prime} \mathrm{E}\right)$ and stored in liquid nitrogen. The voucher specimens were deposited in the Center of Herbarium, Guizhou University of Traditional Chinese Medicine, China, under accession number WB20191003. 
Total DNA was extracted from leaf tissue of $B$. balsamifera using the EZNA Plant DNA extraction kit (OMEGA, USA). Subsequently, DNA purity and quantity were evaluated using NanoPhotometer spectrophotometer (IMPLEN, USA) and Qubit 2.0 Fluorometer (Life Technologies, USA), respectively. A genomic library was constructed using the TruSeq Nano DNA Sample Prep Kit (Illumina, USA), following the manufacturer's protocol. Samples were sequenced on Illumina NovaSeq (Illumina, USA) platform, and 150 bp paired-end reads were generated. The raw reads were deposited in the NCBI sequence read archive with the accession number PRJNA728381.

\section{Chloroplast genome assembly and annotation}

First, the raw reads were filtered using the Trimmomatic (Bolger et al., 2014) software. Next, the cp genome was assembled using the NOVOPlasty (Dierckxsens et al., 2017) software. The Gap Closer (Luo et al., 2012) software was used to repair the inner gaps of the assembly results. Additionally, considering the genome sequence of Pluchea indica (NC_038194.1) as a reference, the whole cp genome of $B$. balsamifera was annotated by DOGMA (Wyman et al., 2004). The structure of transfer RNA (tRNA) genes was determined using the tRNAscan-SE (Lowe and Chan, 2016) online software, and the whole cp genome map was constructed using OGDRAW v.1.2 (Lohse et al., 2007). The cp genome sequence of $B$. balsamifera was uploaded to the GenBank database with the accession number MW769705.

\section{Codon usage analysis and repeat sequence detection}

MEGA v.7.0 (Kumar et al., 2016) was used to analyze the synonymous codon usage and the relative synonymous codon usage (RSCU) of the $B$. balsamifera $\mathrm{cp}$ genome. MISA (Beier et al., 2017) software was used to detect simple repetitive sequences (SSRs) in the cp genome of $B$. balsamifera with the minimum repetitive unit set as follows: single nucleotide $>10$, dinucleotide $>5$, trinucleotide $>4$, tetranucleotide $>3$, pentanucleotide $>3$, and hexanucleotide $>3$. The long repeats in B. balsamifera were identified using the REPuter software (Kurtz et al., 2001). The minimum repeat length was set to 30 $\mathrm{bp}$, and the similarity between repeat sequences was $>90 \%$.

\section{Comparative analysis of chloroplast genomes}

The whole cp genome differences in B. balsamifera were compared with related species; $\mathrm{cp}$ genomes of four Asteraceae plants (Anaphalis sinica, NC_034648.1; Leontopodium leiolepis, NC_027835.1; and Helichrysum italicum, NC_041458.1; P. indica, NC_038194.1) were obtained from GenBank. Additionally, the $m \bar{V}$ ista (Frazer et al., 2004) program with Shuffle-LAGAN mode compared the genome of $B$. balsamifera $\mathrm{cp}$ with those of other selected species.

\section{Phylogenetic analysis}

Cp genomes of 37 Asteraceae plants and three Rosaceae plants were downloaded from the NCBI database, and three Rosaceae plants were set as the outgroup (Table S1). The phylogenetic reconstruction analysis was performed using the RAxML program (Stamatakis, 2014) with the maximum likelihood (ML) method. Sequence comparison was completed using the MAFFT software (Katoh and Standley, 2013), and necessary manual inspection and result adjustment on Se-Al v.2.04 (http://tree.bio.ed.ac.uk/software/). Moreover, GTR + I $+\mathrm{G}$ was selected as the nucleotide substitution model. Finally, the bootstrap values (BS) of each branch of the phylogenetic tree were obtained by 1000 self-expanding repeat analysis.

\section{Results and Discussion}

\section{Chloroplast genome features of $\mathrm{B}$. balsamifera}

The complete cp genome of $B$. balsamifera is $151,170 \mathrm{bp}$ in size, which has a typical quadripartite structure and harbors a pair of inverted repeat regions (IRa and IRb) of 24,982 bp in size, separating the large single-copy (LSC) region of $82,740 \mathrm{bp}$ from the small single-copy (SSC) region of 18,466 bp (Figure 1). The GC content of the complete cp genome, LSC, SSC, and IR regions are $37.50 \%, 35.80 \%, 31.10 \%$, and $43.00 \%$, respectively (Table S2). We found that the GC content in the IR region was the highest, whereas that in the SSC region was the lowest. Related studies have demonstrated that frequent transformation of GC-biased genes in the IR region is the main reason for the high GC content in the IR region (Wu and Chaw, 2015). Overall, 130 genes were annotated, including 85 protein-coding, 37 tRNA, and 8 ribosomal RNA (rRNA) genes (Table S2). Among these genes, 4 rRNA, 7 tRNA, and 6 protein-coding genes were duplicated in the IR region (Table 1). Furthermore, we found that nine protein-coding genes and seven transporter RNA genes contain a single intron and three protein-coding genes have two introns (Table 1). Additionally, rps 12 is a trans-splicing gene with the 5 '-exon located in the LSC region and 3 -end exon duplicated in the IRs, which is common among plant cp genomes.

\section{Codon usage analysis}

Codon bias plays a key role in translation and controls protein production and folding (Quax et al., 2015). This study found that 85 protein-coding genes in $B$. balsamifera genome are encoded by 64 codons; three are stop codons (UAA, UGA, UAG, Table S3). Among the 20 amino acids, leucine (10.65\%) accounts for the largest proportion and cysteine (1.11\%) accounts for the smallest (Figure S1). In other angiosperm $\mathrm{cp}$ genomes, the reported leucine and cysteines are also the most and least abundant amino acids (Tyagi et al., 2020). The frequency of the codon AUU encoding isoleucine is the highest, whereas that of the codon CGC encoding arginine is the lowest (Table S3). Codon usage bias is also measured by calculating the relative synonymous codon usage (RSCU), which represents the ratio between the usage frequency of a specific codon and the expected frequency. If the RSCU value is $>1$, the use frequency of code is higher than the expected frequency, and RSCU value of $<1$ indicates the opposite result (Sharp and Li, 1987). Thirty preferred (RSCU > 1) synonymous codons are detected, indicating that these codons are preferentially used in coding amino acids. Additionally, we found that only the codons encoding Trp (UGG) and Met (AUG) amino acids have no bias (RSCU $=1$ ); however, other codons have an obvious bias in B. balsamifera (Figure S2). Intriguingly, except UUG, all preferentially used codons end 


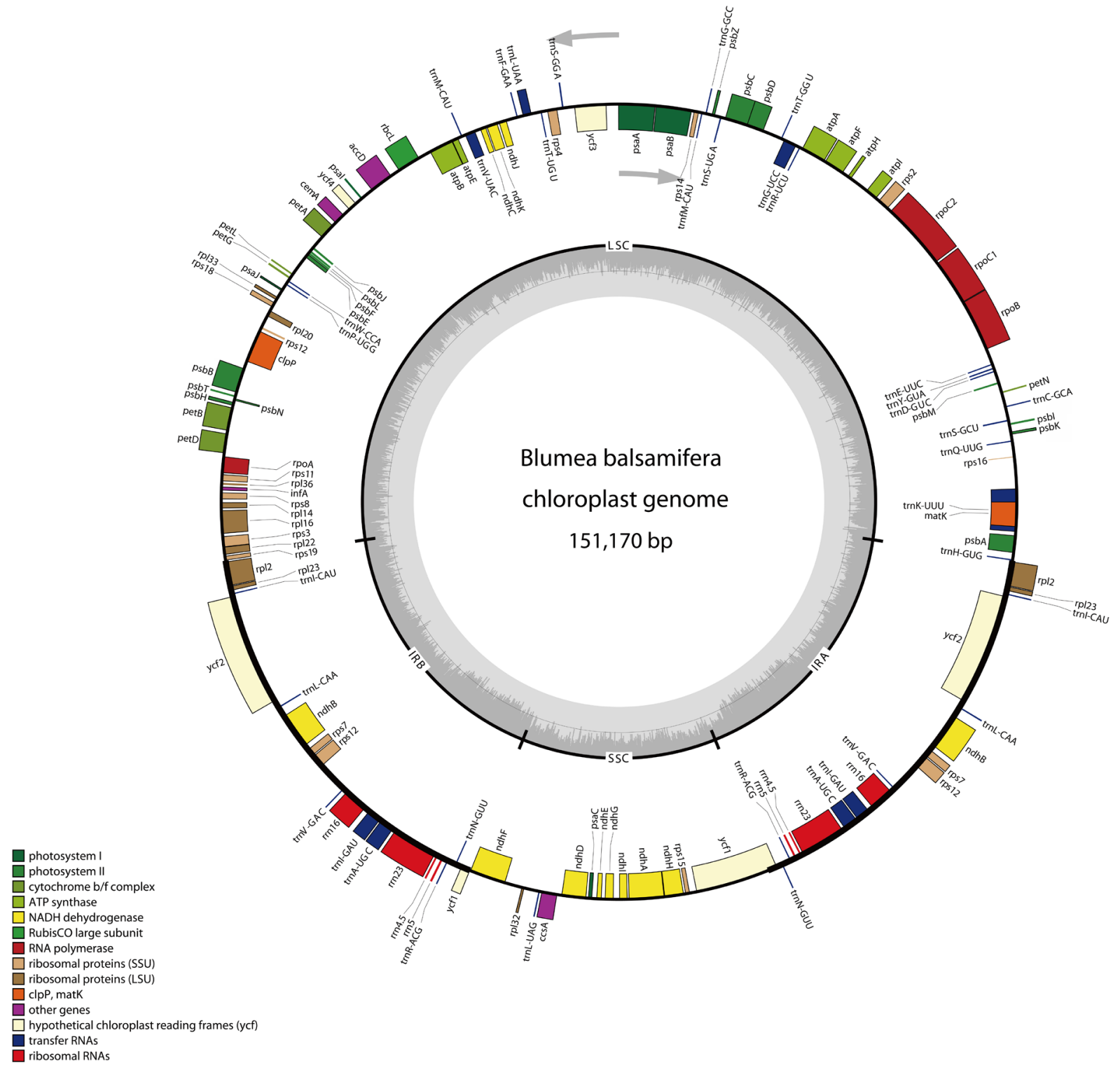

Figure 1 - Gene map of the complete cp genome of Blumea balsamifera. Genes present within the circle are transcribed clockwise and those outside are transcribed counterclockwise. Genes are color-filled based on different functions. Inverted repeat (IR), small single-copy (SSC), and large single-copy (LSC) regions are indicated.

with $\mathrm{A} / \mathrm{U}$. This result agrees with that observed in other species (Munyao et al., 2020; Tyagi et al., 2020), which shows that the dominant codon contains more $A$ or $U$ in codon selection and the high proportion of $\mathrm{A} / \mathrm{U}$ is the driving force of deviation.

\section{Repeat sequence analysis}

Simple sequence repeats (SSRs), known as microsatellite DNA, are molecular markers often used in phylogenetics, identification, and population genetic studies of plant species because they are highly reliable, reproductive, and polymorphic (Kaur et al., 2015). This study identified 53 SSRs in the cp genome of $B$. balsamifera (mononucleotide, dinucleotide, trinucleotide, and tetranucleotide; Table S4). Mononucleotide repeats $(79.37 \%)$ are the most abundant, whereas the trinucleotide repeats $(3.17 \%)$ are the least (Table $\mathrm{S} 4)$, indicating that mononucleotide repeats contribute more to genetic variations than other SSRs. Furthermore, we found that the content of A or T in 4 SSRs was the highest, which illustrates that SSRs usually comprise poly-A and poly-T, rarely tandem guanine $(\mathrm{G})$ and cytosine $(\mathrm{C})$, thereby contributing to the AT richness of the cp genome. Additionally, 40 long repeats are identified in the $B$. balsamifera genome (Table S5), including 18 forward repeats $(\mathrm{F}), 21$ palindromic repeats $(\mathrm{P})$, and one reverse repeat $(\mathrm{R})$. The long repeat sequences range from 30 to $59 \mathrm{bp}$, and most of them are concentrated in the range of 30-49 bp. They mainly exist in the intergenic spacers; however, $p s a B, p s b A, y c f 3, n d h B, y c f 2$, and $y c f 1$ genes comprise the majority that contains long repeats. The long repeats are also detected more in LSC than in SSC and IR regions. Similar results are also noted in other Asteraceae, indicating that long repeats exist in noncoding regions (Tyagi et al., 2020). Overall, repeat sequences can reshape the cp genome and reveal the genetic diversity among different species. 
Table 1 - Genes present in the chloroplast genomes of Blumea balsamifera.

\begin{tabular}{|c|c|c|}
\hline Category & Group of Genes & Name of Genes \\
\hline \multirow[t]{5}{*}{ Self-replication } & Small subunit of ribosome (SSU) & $\begin{array}{c}r p s 2, r p s 3, r p s 4, r p s 7(2), r p s 8, r p s 11, r p s 12^{* *}(2), r p s 14, r p s 15, \\
r p s 16^{*}, r p s 18, r p s 19\end{array}$ \\
\hline & Large subunit of ribosome (LSU) & $r p l 2 *(2), r p l 14, r p l 16 *, r p l 20, r p l 22, r p l 23(2), r p l 32, r p l 33, r p l 36$ \\
\hline & DNA dependent RNA polymerase & rроA, гроB, rроC1*, гроC2 \\
\hline & Ribosomal RNA(rRNA) & $r r n 4.5(2), r r n 5(2), \operatorname{rrn} 16(2), \operatorname{rrn} 23(2)$ \\
\hline & Transfer RNAs (tRNA) & $\begin{array}{l}\operatorname{trn} A-U G C^{*}(2), \operatorname{trn} C-G C A, \operatorname{trn} D-G U C, \operatorname{trn} E-U U C, \operatorname{trn} F-G A A, \\
\operatorname{trnfM}-C A U, \operatorname{trn} G-G C C^{*}, \operatorname{trn} G-U C C^{*}, \operatorname{trn} H-G U G, \operatorname{trn} I-C A U(2), \\
\operatorname{trn} I-G A U^{*}(2), \operatorname{trn} K-U U U^{*}, \operatorname{trn} L-C A A(2), \operatorname{trn} L-U A A^{*}, \operatorname{trn} L-U A G, \\
\operatorname{trn} M-C A U, \operatorname{trn} N-G U U(2), \operatorname{trn} P-U G G, \operatorname{trn} Q-U U G, \operatorname{trn} R-A C G(2), \\
\operatorname{trn} R-U C U, \operatorname{trn} S-G C U, \operatorname{trn} S-G G A, \operatorname{trn} S-U G A, \operatorname{trn} T-G G U, \operatorname{trn} T-U G U, \\
\operatorname{trn} V-G A C^{*}(2), \operatorname{trn} V-U A C, \operatorname{trn} W-C C A, \operatorname{trn} Y-G U A\end{array}$ \\
\hline \multirow[t]{6}{*}{ Photosynthesis } & Photosystem I & psaA, psaB, psaC, psaI, psaJ \\
\hline & Photosystem II & $\begin{array}{c}p s b A, p s b B, p s b C, p s b D, p s b E, p s b F, p s b H, p s b I, p s b J, p s b K, p s b L, \\
p s b M, p s b N, p s b T, p s b Z\end{array}$ \\
\hline & Subunits of NADH-dehydrogenase & $n d h A^{*}, n d h B^{*}(2), n d h C, n d h D, \underset{n d h E, n d h F, n d h G, n d h H, n d h I, n d h J,}{n d h K}$ \\
\hline & Subunits of cytochrome $b / f$ complex & pet $A, \operatorname{pet} B^{*}, \operatorname{pet} D^{*}, \operatorname{pet} G, \operatorname{pet} L, \operatorname{pet} N$ \\
\hline & Subunits of ATP synthase & atp $A$, atpB, atpE, atpF*, atpH, atpI \\
\hline & Large subunit of rubisco & $r b c L$ \\
\hline \multirow[t]{6}{*}{ Other genes } & ATP-dependent protease subunit $p$ gene & $\operatorname{clp} P * *$ \\
\hline & Translational initiation factor & $\inf A$ \\
\hline & Maturase & $\operatorname{mat} K$ \\
\hline & Envelope membrane protein & сетA \\
\hline & Subunit of acetyl-CoA-carboxylase & $a c c D$ \\
\hline & C-type cytochrome synthesis gene & $\operatorname{ccs} A$ \\
\hline Unknown function & Hypothetical chloroplast reading frames (ycf) & $y c f 1, y c f 2(2), y c f 3^{* *}, y c f 4$ \\
\hline
\end{tabular}

(2) indicates the number of the repeat unit is 2 ; *genes with one intron; **genes with two introns.

\section{Comparative genome analysis}

Comparative analysis of DNA sequences helps in identifying important gene regions and functional genes. The mVISTA is a common tool for comparative genome analysis and helps in quickly identifying the conserved regions of DNA sequences (Ratnere and Dubchak, 2009). By comparing the whole cp genome of $B$. balsamifera and other four Asteraceae plants (A. sinica, L. leiolepis, H. italicum, and $P$. indica), we found that the conservation of the IR region among the five species was higher than that of the LSC and SSC regions. Genetic variability was mainly concentrated in the intergenic or noncoding region (Figure 2). The coding regions with a large variation in the five chloroplast genomes are $m a t K, a t p B$, accD , cemA, rpoA, ycf2, ndhF, ccs $A, n d h I, n d h H, r p s 15$, and $y c f 1$ (Figure 2). The regions with large differences are widely used to study population and plant system genetics (Kim and Jansen, 1995). For example, mat $K$ has been widely used in the core universal DNA barcode of species, whereas $y c f l$ is used in plant phylogeny and DNA barcode research (Dong et al., 2015; Yang et al., 2017). Additionally, accD, rpoA, ccsA, and $n d h F$ have significant differences, which can be used to study plant development systems (Li et al., 2019). We can study these regions further to develop more DNA barcode markers for the identification of Blumea species.

\section{Phylogenetic analysis}

With the rapid development of sequence technology, several cp genomes of plants have been revealed, making it possible to explore the relationship between plant phylogeny and evolution from the molecular perspective (Tonti-Filippini et al., 2017). For the determination of the phylogenetic status and evolutionary relationship of B. balsamiferae in Asteraceae, the complete cp genome sequences of 37 reported Asteraceae species were selected to construct the maximum likelihood (ML) phylogenetic tree and three species of the Rosaceae family were considered as the outgroup (Figure 3). Phylogenetic analysis shows that Asteraceae species form a monophyletic group and are divided into several subgroups (Anthemideae, Astereae, Gnaphalieae, Senecioneae, Heliantheae alliance, Inuleae, Cardueae, and Mutisieae). $B$. balsamifera and $P$. indica are clustered into the same branch with a bootstrap value of $100 \%$, and both belonged to Inuleae. The genetic relationship between Inuleae and Heliantheae alliance is close, whereas that between Inuleae and Astereae is far, which is consistent with the results of Tyagi et al. (2020). Our classification supports Anderberg's 1991 and 2005 classification results from a genomic perspective. This study fills the gap in the research of the cp genome of Blumea plants, which provides abundant information regarding the taxonomic study of this genus in Asteraceae plants. 
Pluchea indica

Anaphalis sinica

Helichrysum italicum

Leontopodium leiolepis

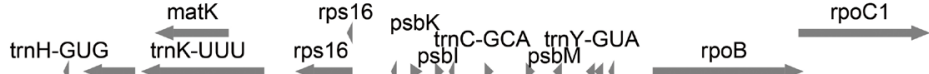

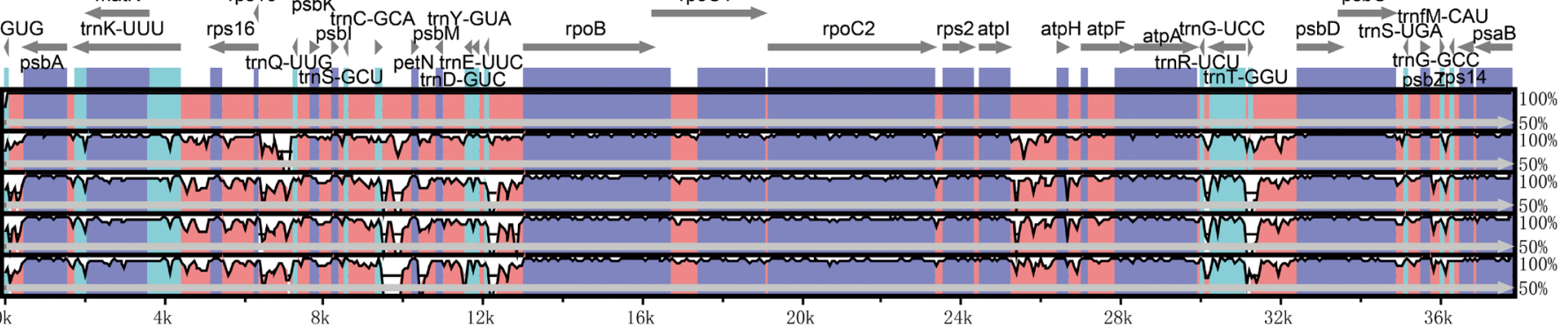

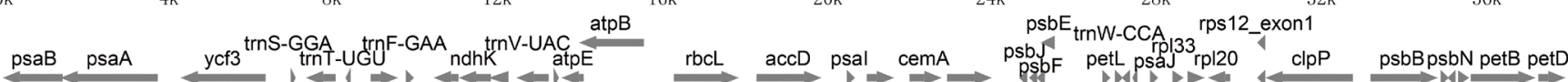

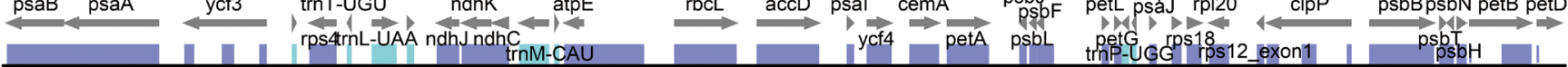
(10k

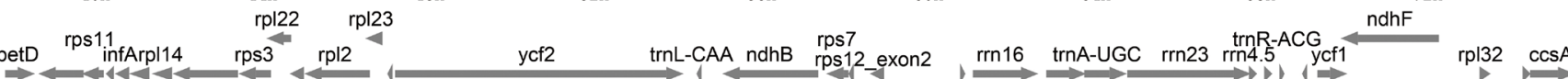

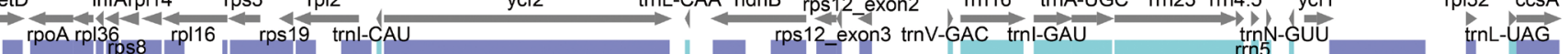

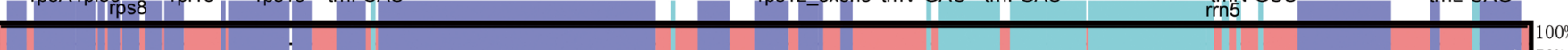
The एक

contig
gene
exon
UTR
CNS
CNS ${ }_{76}$

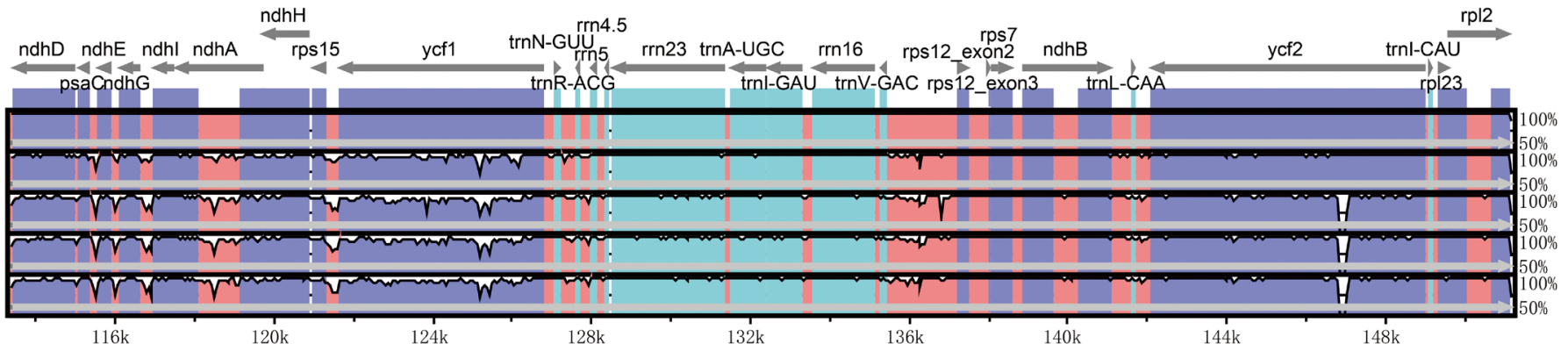

Figure 2 - The cp genomes of Blumea balsamifera and four other Asteraceae plants were compared using the mVista program with Blumea balsamifera as the reference. The arrow indicates the position and orientation f the gene. The Y-axis represents the percentage of identity among genome sequences $(50 \%-100 \%)$ 


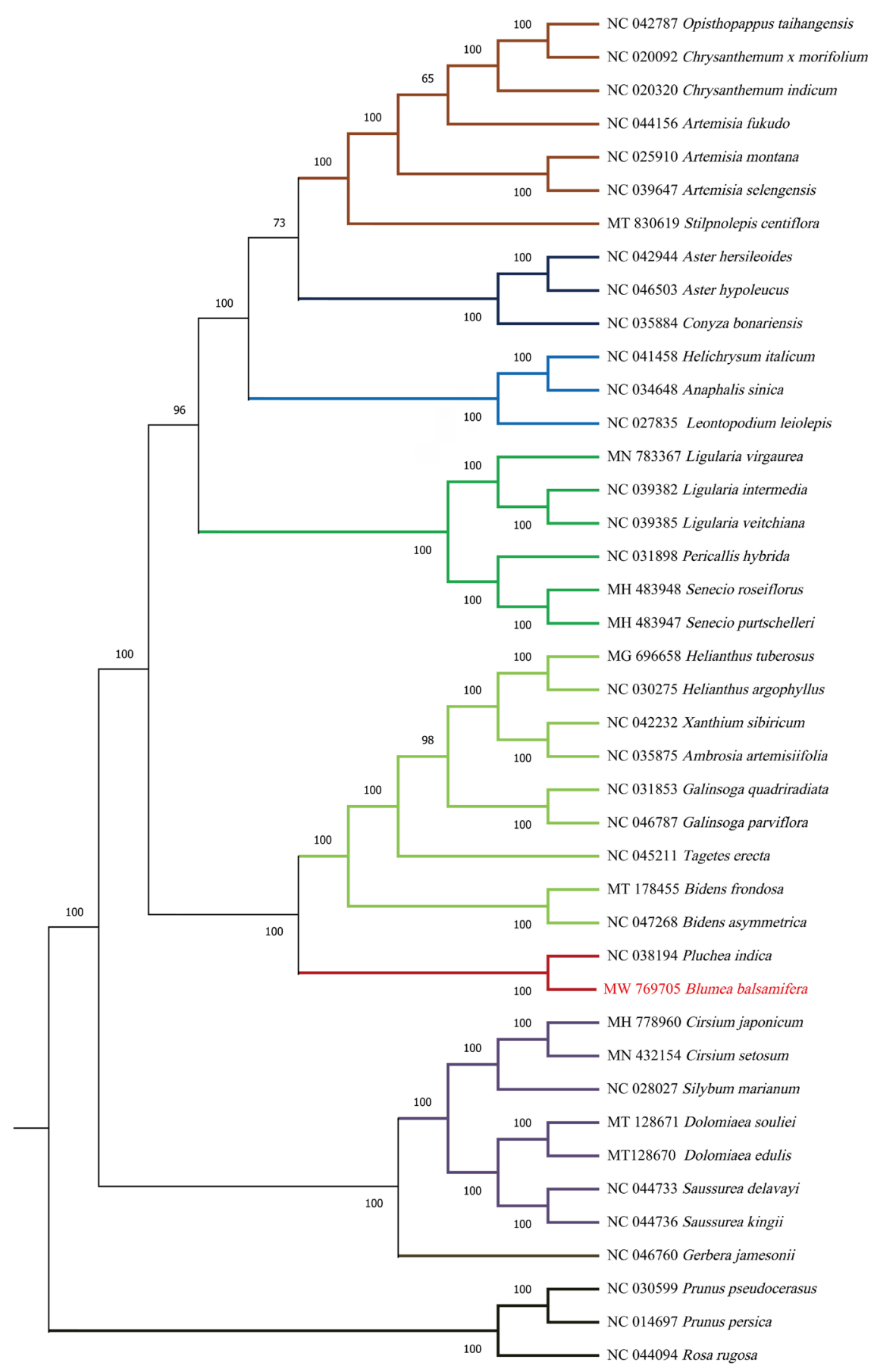

Anthemideae

Astereae

Gnaphalieae

Senecioneae

Heliantheae alliance

Inuleae

Cardueae

Mutisieae

Out

Figure 3 - The maximum likelihood (ML) phylogenetic tree based on the complete chloroplast genome sequence was constructed with three species of Rosaceae as outgroup. The number above the tree node indicates the bootstrap support value.

\section{Conclusions}

$\mathrm{Cp}$ genomes have been widely considered an informative and valuable resource for molecular marker development and phylogenetic reconstruction in plant species. In this study, the complete cp genome of $B$. balsamifera was reported for the first time. The cp genome of $B$. balsamifera is 151,170 bp in size, and its genome structure, gene number, and gene sequence are similar to those of other Asteraceae plants. We found that there are more $\mathrm{A}$ or $\mathrm{U}$ in the preferred codon. Combining the results with previous studies, we speculate that the high content of $\mathrm{A}$ or $\mathrm{U}$ is an important reason for the deviation of coding genes. Furthermore, 53 SSRs were found, which can be used for studies on population genetics and genetic breeding of Blumea. Comparison of the whole cp genome of $B$. balsamifera with that of other Asteraceae species indicates that the IR region is more conservative than the SSC and LSC regions. Finally, a phylogenetic tree was constructed based on the complete chloroplast genomes of 37 Asteraceae species and three Rosaceae species, confirming that $B$. balsamifera had the closest relationship with $P$. indica from the molecular viewpoint and revealed the position of B. balsamifera in Asteraceae. Thus, the complete cp genome 
of $B$. balsamifera provides valuable genetic information for this genus and lays a foundation for identifying and studying population evolution in Asteraceae species.

\section{Acknowledgments}

This work was supported by the National Natural Science Foundation of China (No. 81560707), First class discipline construction project in Guizhou Province (GNYL[2017]008), Project of Engineering Research Center of Guizhou University (KY[2017]018), Excellent young scientific and Technological Talents Project of Guizhou Province ([2019]5658).

\section{Conflict of Interest}

The authors declare no conflict of interest.

\section{Author Contributions}

$\mathrm{CZ}, \mathrm{WFX}$, and $\mathrm{YH}$. conceived and designed the experiments. Materials were collected by BW and QWS; Formal analysis was performed by $\mathrm{WFX}$ and $\mathrm{YH}$; $\mathrm{CZ}$ analyzed the data and wrote the manuscript; revision and manuscript editing was done by CLC and QYC; All authors have read and agreed to the published version of the manuscript.

\section{References}

Anderberg AA (1991) Taxonomy and phylogeny of the tribe Inuleae (Asteraceae). Plant Syst Evol 176:75-123.

Anderberg AA, Eldenäs P, Bayer R and Englund M (2005) Evolutionary relationships in the Asteraceae tribe Inuleae (incl. Plucheeae) evidenced by DNA sequences of ndhF; with notes on the systematic positions of some aberrant genera. Org Divers Evol 5:135-146.

Beier S, Thiel T, Münch T, Scholz U and Mascher M (2017) MISAweb: a web server for microsatellite prediction. Bioinformatics 33:2583-2585.

Bolger AM, Lohse M and Usadel B (2014) Trimmomatic: a flexible trimmer for Illumina sequence data. Bioinformatics 30:21142120.

Chinese Academy of Sciences editorial commission of the flora (1988) Flora Republicae Popularis Sinicae, Sciences Press, Beijing, v. 75, 19 pp.

Dempewolf H, Rieseberg LH and Cronk QC (2008) Crop domestication in the Compositae: a family-wide trait assessment. Genet Resour Crop Evol 55:1141-1157.

Dewen Q (2005) Chinese Herbal Medicine-Miao Medicine Roll. Guizhou Science and Technology Publishing House, Guiyang, $265 \mathrm{pp}$.

Dierckxsens N, Mardulyn P and Smits G (2017) NOVOPlasty: de novo assembly of organelle genomes from whole genome data. Nucleic Acids Res 45:e18.

Dong W, Xu C, Li C, Sun J, Zuo Y, Shi S, Cheng T, Guo J and Zhou S (2015) ycfl, the most promising plastid DNA barcode of land plants. Sci Rep 5:8348.

Frazer KA, Pachter L, Poliakov A, Rubin EM and Dubchak I (2004) VISTA: computational tools for comparative genomics. Nucleic Acids Res 32:W273-9.

Hasegawa H, Yamada Y, Komiyama K, Hayashi M, Ishibashi M, Yoshida T, Sakai T, Koyano T, Kam TS, Murata K et al. (2006) Dihydroflavonol BB-1, an extract of natural plant Blumea balsamifera, abrogates TRAIL resistance in leukemia cells. Blood 107:679-688.

Katoh K and Standley DM (2013) MAFFT multiple sequence alignment software version 7: Improvements in performance and usability. Mol Biol Evol 30:772-780.
Kaur S, Panesar PS, Bera MB and Kaur V (2015) Simple sequence repeat markers in genetic divergence and marker-assisted selection of rice cultivars: A review. Crit Rev Food Sci Nutr 55:41-49.

Kim KJ and Jansen RK (1995) ndhF sequence evolution and the major clades in the sunflower family. Proc Natl Acad Sci U S A 92:10379-83.

Kumar S, Stecher G and Tamura K (2016) MEGA7: Molecular Evolutionary Genetics Analysis Version 7.0 for bigger datasets. Mol Biol Evol 33:1870-1874.

Kurtz S, Choudhuri JV, Ohlebusch E, Schleiermacher C, Stoye J and Giegerich R (2001) REPuter: The manifold applications of repeat analysis on a genomic scale. Nucleic Acids Res 29:4633-4642

Li Y, Jia LK, Wang ZH, Xing R, Chi XF, Chen SL and Gao QB (2019) The complete chloroplast genome of Saxifraga sinomontana (Saxifragaceae) and comparative analysis with other Saxifragaceae species. Braz J Bot 42:601-611.

Lohse M, Drechsel O and Bock R (2007) OrganellarGenomeDRAW (OGDRAW): a tool for the easy generation of high-quality custom graphical maps of plastid and mitochondrial genomes. Curr Genet 52:267-274.

Lowe TM and Chan PP (2016) tRNAscan-SE On-line: integrating search and context for analysis of transfer RNA genes. Nucleic Acids Res 44:W54-7.

Luo R, Liu B, Xie Y, Li Z, Huang W, Yuan J, He G, Chen Y, Pan $\mathrm{Q}$ and Liu Y et al. (2012) SOAPdenovo2: an empirically improved memory-efficient short-read de novo assembler. Gigascience 1:18.

Munyao JN, Dong X, Yang JX, Mbandi EM, Wanga VO, Oulo MA, Saina JK, Musili PM and Hu GW (2020) Complete chloroplast genomes of Chlorophytum comosum and Chlorophytum gallabatense: genome structures, comparative and phylogenetic analysis. Plants (Basel) 9:296.

Quax TE, Claassens NJ, Söll D and van der Oost J (2015) Codon bias as a means to fine-tune gene expression. Mol Cell 59:149-161.

Ratnere I and Dubchak I (2009) Obtaining comparative genomic data with the VISTA family of computational tools. Curr Protoc Bioinformatics 10:Unit 10.6.

Rodríguez-Ezpeleta N, Brinkmann H, Burey SC, Roure B, Burger G, Löffelhardt W, Bohnert HJ, Philippe H and Lang BF (2005) Monophyly of primary photosynthetic eukaryotes: green plants, red algae, and glaucophytes. Curr Biol 15:1325-1330.

Sakee U, Maneerat S, Cushnie TP and De-Eknamkul W (2011) Antimicrobial activity of Blumea balsamifera (Lin.) DC. extracts and essential oil. Nat Prod Res 25:1849-1856.

Sharp PM and Li WH (1987) The codon Adaptation Index--a measure of directional synonymous codon usage bias, and its potential applications. Nucleic Acids Res 15:1281-1295.

Smith DR (2015) Mutation rates in plastid genomes: they are lower than you might think. Genome Biol Evol 7:1227-1234.

Stamatakis A (2014) RAxML version 8: a tool for phylogenetic analysis and post-analysis of large phylogenies. Bioinformatics 30:1312-1313.

Tonti-Filippini J, Nevill PG, Dixon K and Small I (2017) What can we do with 1000 plastid genomes? Plant J 90:808-818.

Toralba JV, Quiming NS and Palacpac JS (2015) RP-HPLC analysis of quercetin in the extract of sambong (Blumea balsamifera (L) DC) leaves. Science Diliman 27:48-63.

Tyagi S, Jung JA, Kim JS and Won SY (2020) Comparative analysis of the complete chloroplast genome of mainland Aster spathulifolius and other Aster species. Plants (Basel) 9:568.

Wicke S, Schneeweiss GM, dePamphilis CW, Müller KF and Quandt D (2011) The evolution of the plastid chromosome in land plants: gene content, gene order, gene function. Plant Mol Biol 76:273-297. 
Wu CS and Chaw SM (2015) Evolutionary stasis in cycad plastomes and the first case of plastome GC-biased gene conversion. Genome Biol Evol 7:2000-2009.

Wyman SK, Jansen RK and Boore JL (2004) Automatic annotation of organellar genomes with DOGMA. Bioinformatics 20:32523255.

Xu J, Jin DQ, Liu C, Xie C, Guo Y and Fang L (2012) Isolation, characterization, and NO inhibitory activities of sesquiterpenes from Blumea balsamifera. J Agric Food Chem 60:8051-8058.

Yang J, Vázquez L, Chen X, Li H, Zhang H, Liu Z and Zhao G (2017)

Development of chloroplast and nuclear DNA markers for Chinese oaks (Quercus Subgenus Quercus) and assessment of their utility as DNA barcodes. Front Plant Sci 8:816.

\section{Supplementary material}

The online material available for this article contains the following:

Table S1 - List of 40 chloroplast genomes used in the phylogenetic analysis
Table S2 - Features of the chloroplast genomes of Blumea balsamifera

Table S3 - Codon usage in the Blumea balsamifera chloroplast genomes

Table S4 - Types and amount of SSRs of Blumea balsamifera Table S5 - Analysis of long sequence repeats in the Blumea balsamifera chloroplast genome

Figure S1 - Ratio of amino acids and stop codons in the cp genome of Blumea balsamifera

Figure S2 - RSCU histogram of Blumea balsamifera. The upper figure shows the sum of RSCU values of codons in amino acids. The following blocks represent all codons that encode each amino acid.

Associate Editor: Ana Tereza Vasconcelos

License information: This is an open-access article distributed under the terms of the Creative Commons Attribution License (type CC-BY), which permits unrestricted use, istribution and reproduction in any medium, provided the original article is properly cited. 